\section{P5-S6.08 STD SURVEILLANCE AND CONTROL: DO STD AND FAMILY PLANNING CLINICS MATTER?}

doi:10.1136/sextrans-2011-050108.565

S Doshi, K Owusu-Edusei. Centers for Disease Control and Prevention, Atlanta, USA

Background Information on the potential impact of the presence of an STD and/or family planning (FP) clinic on STD surveillance and control is scant. In the study, we examine the impact of STD and/or publicly funded Title V, X, and XX FP clinics on county-level surveillance and control of three reportable STDs (chlamydia (CT), gonorrhoea (GC) and primary and secondary syphilis (P\&S)) in Texas during 2000 and 2007. Specifically, we examined the following two questions: (1) Are the counties with STD/FP clinics reporting relatively more cases of STDs? (2) Does having STD service(s) (the presence of an STD/FP clinic) in a county matter?

Methods We used spatial regression to analyse the impact of STD/ FP clinics on county-level STD morbidity using surveillance data on CT, GC, and P\&S. the impact of STD/FP clinics on STD control was examined using a backward stepwise regression on the changes in incidence rates between 2000 and 2007. Dummy variables represented the presence of STD/FP clinic(s). Incidence rates were transformed into natural logs. County-level demographics for 2000 and 2007 were used as control variables.

Results The coefficients on the dummy variables representing the presence of STD/FP clinics were: 0.11 ( $95 \%$ CI 0.05 to $0.16, \mathrm{p}<0.01$ ) in 2000 and 0.07 (CI 0.02 to $0.12, \mathrm{p}<0.01$ ) in 2007 for CT; 0.13 (CI 0.05 to $0.20, \mathrm{p}<0.01)$ in 2000 and 0.06 (CI -0.002 to $0.12, \mathrm{p}<0.01)$ in 2007 for GC and 0.18 (CI 0.12 to $0.23, p<0.01$ ) in 2000 for P\&S (2007 was not significant). This implies that the transformed GC and CT rates in the counties with STD/FP clinics increased by at least $6 \%$ in 2000 and 2007, while P\&S increased by $18 \%$ in 2000 . The coefficient for the changes in rates between 2000 and 2007 were: -0.04 (CI 0.01 to $-0.09, \mathrm{p}<0.10$ ) for CT; -0.08 (CI -0.02 to -0.14 , $\mathrm{p}<0.01$ ) for GC and -0.09 (CI -0.03 to $-0.15, \mathrm{p}<0.01$ ) for $\mathrm{P} \& \mathrm{~S}$. Thus, the transformed incidence rates of $\mathrm{GC}$ and $\mathrm{P} \& \mathrm{~S}$ reduced by $8 \%$ and $9 \%$, respectively, between 2000 and 2007 for those counties that had at least one STD/FP clinic.

Conclusion The results from this ecological study are consistent with (but do not establish) a causal relationship between having an STD/FP clinic and improved surveillance and/or reduction in STDs at the county-level in Texas. However, the results suggest that STD/ FP clinics play an important role in STD surveillance and control. Finer level analyses (such as census block or cities) may be able to establish a strong causal relationship.

\section{P5-S6.09 USING SYNDROMIC MANAGEMENT OF STI AS AN ENTRY POINT TO HIV PREVENTION INTERVENTION IN A RESOURCE POOR SETTING}

doi:10.1136/sextrans-2011-050108.566

\section{Bassey, A Eyo. ECEWS, Uyo, Nigeria}

Issue Almost all the patients who presents to the hospital with symptoms and/or signs of sexually transmitted infection have been engaged with unprotected sexual intercourse, thus they are predispose to contacting STI including HIV and complications. STIs can increase the risk of HIV acquisition and transmission by a factor of up to 10 . The WHO estimates that more than three million new curable STI infections occur annually among people aged 15-49 worldwide. In 1995, over half of female patients seeking treatment for a sexually transmitted infection (STI) tested in 13 states were HIV positive. Among STI patients in 10 states, in 2000, HIV prevalence increased by age, the lowest rate was ages 10-19, and the highest among those ages 50+. Akwa Ibom State has one of the highest rates among selected states for 2000 with $21 \%$ of STI patients tested HIV positive In 2008, Akwa Ibom and Cross River States had the highest rate of HIV infection in the South South Zone at $9.7 \%$ and $6 \%$ respectively. The health seeking behaviour of the poor at public health facilities is influenced by cost, the fear that services are not going to be confidential and the fear of meeting people they know at the healthcare facility. Thus this sexual risky population often do not always access care, thus contributing to the underserved most at risk population in the community.

Method ECEWS implemented Condom and Other Prevention using the combination prevention among the probable STI patients in rural health facility and missionary health centres in Akwa Ibom and Cross River state using STI counselling, $\mathrm{ABC}$ messages, risk reduction, condom promotion, HTC referral, syndromic diagnosis and treatment of STI. Drugs were provided and dispensed free according to the syndromic management guidelines of $\mathrm{WHO} / \mathrm{FMOH}$. Preceding this, communities were mapped as areas of high sexual activity and STI prevalence. The CHEW, nurses and doctors in those facilities were selected and trained as health educators to reach this people with HIV prevention messages and syndromic management of STI.

Result 13401 probable STI patients were reached with risk reduction counselling for HIV, condom promotion and STI management by 35 ECEWS trained health educators across primary health centres, missionary hospitals and other rural health post across 20 sites in Akwa Ibom and Cross River states. The strengthened referral system enable majority (88\%) of the client to access HTC with little or no challenge. A HIV positivity rate of $12 \%$ was observed among this population.

Conclusion Using syndromic management as an entry point, poor and most at risk individual in poor rural settings easily accessed effective of HIV prevention messages, counselling, and treatment of STI and behavioural plan towards sexual prevention of HIV. Therefore, syndromic management of STI provides cure and ample opportunity as entry point to reach this MARP in a resource poor setting where laboratory investigation is not easily accessible in intervention of sexual prevention of HIV/AIDS.

\section{P5-S6.10 ABSTRACT WITHDRAWN}

\section{P5-S6.11 HIV PREVENTION WITH POSITIVES PROVIDER TRAINING IN A RESOURCE-CONSTRAINED SETTING: THE US VIRGIN ISLANDS}

doi:10.1136/sextrans-2011-050108.567

${ }^{1} \mathrm{~A}$ Canete, ${ }^{1} \mathrm{G}$ Nagendra, ${ }^{2} \mathrm{E}$ Chun, ${ }^{3} \mathrm{R}$ Olans, ${ }^{4} \mathrm{~T}$ Ciprian, ${ }^{5} \mathrm{~S}$ Dreisbach. ${ }^{1}$ Public Health Solutions, New York, USA; ${ }^{2}$ NYC Department of Health \& Mental Hygiene, New York, USA; ${ }^{3}$ US Virgin Islands Department of Health, USA; ${ }^{4}$ NYC Department of Health \& Mental Hygiene, New York, USA; ${ }^{5}$ University of Colorado, USA

Background To describe HIV Prevention with Positives training for healthcare providers in the USA Virgin Islands (USVI).

Methods The Caribbean region has the world's second highest adult HIV prevalence after Africa. In 2007, the prevalence rate of HIV among adults and adolescents in the USVI was 641.3 per 100000 population. In the USVI, HIV clinical care is provided by three physicians at the USVI Department of Health or at community health centers. Due to a shortage of trained clinicians, non-clinical providers play a crucial role in the delivery of comprehensive HIV care. Ask Screen Intervene (ASI), a training curriculum, was developed by the National Network of STD/HIV Prevention Training Centers and the AIDS Education and Training Centers with support from Centers of Disease Control and Prevention. ASI was designed for clinical HIV providers and discusses risk assessment, STD screening, prevention messages and partner services for HIV+ patients. In June 2010, the Region II STD/HIV Prevention Training Center offered ASI in the USVI, in St. Croix and St. Thomas. ASI 